\title{
AN IN VITRO COMPARISON OF LINEAR MEASUREMENTS OF PANORAMIC VIEWS ACQUIRED VIA 2D OR 3D IMAGING
}

\author{
Farid Medhat Farid* and Ghada Borhan*
}

\begin{abstract}
Introduction: Panoramic radiography and $\mathrm{CBCT}$ are common imaging techniques in general dental practice. Some clinicians still refer patients for additional conventional digital panoramic radiographs despite the fact that $\mathrm{CBCT}$ data has already been acquired. The purpose of this study was to evaluate the efficacy of Digital panoramic radiographic images derived from conventional and CBCT generated methods, regarding their linear measurements' accuracy in different anatomical sites in dry human mandibles compared to real measurements obtained by a digital vernier caliper.

Materials and Methods: Measurements from five adult human dry mandibles were made separately on the right and left sides ending up with ten evaluated samples. Several linear measurements related to mental and mandibular foramina were obtained. All were compared to real measurements.
\end{abstract}

Results: Cronbach's alpha inter- and intra- observer agreement in all modalities used ranged from "very good to perfect". No statistically significant difference between linear measurements' means in all modalities used when compared to real ones. Only two measurements; one was significantly underestimated in all modalities, the other was significantly overestimated in manual reformatted panoramic.

Conclusion: Comparable results were shown when linear measurements' means from Digital panoramic, CBCT derived Auto and Manual reformatted panoramic were compared to real ones. Radiation dose to patients must be concerned.

\section{INTRODUCTION}

Radiographs are an important part of dental diagnosis and treatment planning. Radiographs are often used in many branches of dentistry to assess vertical linear measurements (Wakoh et al., 2006). Images generated must allow acquiring measurements that can reproduce the real conditions (Langlois et al., 2011).

Panoramic radiography is a common imaging technique in general dental practice as it provides a view for teeth and the surrounding structures. Millions of these radiographs are taken annually

* Lecturer in the Oral Radiology Department in Cairo University 
for diagnostic (Devlin et al., 2008) and treatment purposes (Park, 2010).

The major disadvantages of panoramic radiography are an unpredictable distortion of the visualized structures and the low level of reproducibility. Magnification in panoramic radiographs occurs in both vertical and horizontal directions and varies considerably (1.1-1.7 times) (Siu et al., 2010).

Measurement of mandibular anatomy is problematic when using panoramic radiography. Previous studies suggest that projection geometry, focal plane shape, differential vertical and horizontal magnification factors, and operator error in patient positioning affect the utility of panoramic images to provide accurate measurements (Ludlow et al., 2007).

Cone beam computed tomography images experienced substantial development in dentistry during the past decade. It is now a diagnostic tool used worldwide as an auxiliary for complete diagnosis in many clinical challenges (Guerrero et al., 2006 and Nakagawa et al., 2002). Complex computer algorithms are required to convert 3D medical and cone beam CT volumes to images that simulate 2D panoramic radiographs, by removing all voxels information that lie outside the specified focal trough. Panoramic reconstruction from the $\mathrm{CT}$ data improves image clarity by reducing geometric distortions, blurring and artifacts from superimpositions (Tohnak et al., 2006). CBCT images are reformatted slices of the maxilla and mandible; they are free of magnification, superimposition of the neighboring structures, and the other problems inherent to panoramic radiography (Angelopoulos et al.,2008). Head position can be standardized after patient scans are complete and focal trough can be customized based on tooth position and angulation. Reconstructed images can remove many of the distortion variables inherent in the traditional panoramic images (Hutchinson et al., 2005).
Reformatted panoramic views from some CBCT scans may be able to offer equal diagnostic quality compared with the digital panoramic images commonly used in dental practices (Pittayapat et al., 2013).

Some clinicians still refer patients for additional conventional digital panoramic radiographs and conventional lateral cephalograms despite the fact that CBCT data has already been acquired. If the panoramic images generated from threedimensional data have equal diagnostic quality as conventional digital panoramic radiographs, then it is not necessary for clinicians to take extra conventional 2D radiographs, thus patients' datasets will be more compact and the radiation dose to patients can be reduced. There is only little evidence that can prove if the panoramic images generated from three dimensional data (CT or CBCT) have equal diagnostic quality as conventional digital panoramic radiographs (Pawelzik et al., 2002 and Angelopoulos et al., 2008).

The purpose of this study was to evaluate the efficacy of digital panoramic and CBCT-derived Auto reformatted panoramic (the panoramic curve is automatically defined by Romexis® software) and CBCT-derived Manual reformatted panoramic (the panoramic curve is defined by the operator and then the new panoramic view will be automatically calculated by the Romexis ${ }^{\circledR}$ software), regarding their linear measurements' accuracy in different anatomical sites in dry human mandibles compared to real measurements obtained by a digital vernier caliper.

\section{MATERIALS AND METHODS}

Five adult human dry mandibles of unknown age or gender were included in the present study. They were obtained from the department of Anatomy, School of Medicine Cairo University. Measurements were made separately on the right and left mandibular sides ending up with ten evaluated samples. 
In order to simulate a clinical situation, no radio-opaque markers were used, as suggested by Kositbowornchai et al. (2007) they were measured directly. According to Kobayashi et al. (2004) and Mischkowski et al. (2007) when anatomical structures are used as landmarks, their correct identification might be a problem when estimating the actual distances between them. However, it might be argued that such an approach is more clinically relevant than making measurements between welldefined structures in an artificial object.

The radio-opaque markers were only used to verify the correct sites of the mental and mandibular foramina on radiographs. Gutta Percha of size 40 were attached vertically to the desired foramina using clear adhesive tape. Paper adhesive labels were placed to identify each mandible by a number.

In order to simulate soft tissues, the mandibles' outer surfaces were covered by $11 \mathrm{~mm}$ of Cavex ${ }^{\circledR}$ pink set up modeling wax (Cavex Holland BV, Haarlem, The Netherlands) (Schropp et al., 2012).

\section{Imaging Procedures}

For both panoramic and CBCT imaging the mandibles were positioned according to the protocol established by the manufacturer with the aid of light beam markers placed on equivalent patient landmarks.

Panoramic imaging was carried out using Planmeca Proline XC (Planmeca Oy, Helsinki, Finland) with standardized settings $(60 \mathrm{kV}, 10 \mathrm{~mA}$ and 18.0 s). Automatic spine compensation (ASC) feature in panoramic machines automatically adjust $\mathrm{kV}$ (by a slight increase) at the area of the spine to counteract for its presence. Thus exposure parameters of panoramic radiography in our study were reduced to 60 Kvp, thus avoiding burn out of that area in the resultant radiographs. Exposure parameters were the same in all panoramic radiographs.

Planmeca Proline XC provides nine different combinations of sizes \& shapes to adjust the focal trough to fit the patients' arch form. In our study we chose the most appropriate one, as according to $A L-N a k i b$, (2005) the size \& shape of the human jaw varies considerably depending on the patient's size, race \& sex. So it is better to have forms of the focal trough that are adjustable \& can be selected to correspond to the patients' anatomy \& the object to be radiographed.

Mandibles were fixed to the panoramic machine using the bite-guide. Before image recording, a Hex jam nut of known height $6.86 \mathrm{~mm}$, obtained by the digital vernier caliper, was fixed to the mandibles' outer surfaces using clear adhesive tape in order to achieve calibration and standardization of measurements. The 1:1 button on software showed the hex jam nut to be $7.01 \mathrm{~mm}$ while the actual hex jam nut height as obtained by the digital vernier caliper was $6.86 \mathrm{~mm}$. Thus calibrating button in the software was pressed and the hex jam nut was measured on the software which showed 91 pixels. The software asked for the coinciding measurement in $\mathrm{mm}$ which was previously determined by the digital vernier caliper as $6.86 \mathrm{~mm}$, and then the software completed the calibration process. According to Schropp et al. (2009) magnification can be adjusted in digital panoramic radiography with a reference ball calibration method that allows 1:1 image visualization on the screen and the use of software-based measurement tools.

The same Hex jam nut was used in all digital panoramic radiographic examinations. The upper and lower surfaces of the hex jam nut were marked using a black pen to ensure that the same surfaces were used in the same direction in each time they were used.

CBCT imaging was carried out using the same CBCT unit Planmeca ProMax ${ }^{\circledR}$ 3D Classic (Planmeca Oy, Helsinki, Finland). The first, second and third mandibles were imaged under an electric regimen of $68 \mathrm{kV}$ and $7 \mathrm{~mA}$. The forth mandible was imaged under an electric regimen of $62 \mathrm{kV}$ 
and $5 \mathrm{~mA}$. The fifth mandible was imaged under an electric regimen of $64 \mathrm{kV}$ and $6 \mathrm{~mA}$. Cadaver mandibles were fixed with a horizontal plate to the Planmeca ProMax ${ }^{\circledR}$ 3D Classic CBCT device (Planmeca Oy, Helsinki, Finland). No calibration was performed. CBCT generates reformatted images of panoramic and cross-sectional views of the jaws in real size (1:1) (Guerrero et al., 2006 and Scarfe et al.,2006). The defined distances were measured utilizing the measurement's tools of the given software.

CBCT radiographic measurements included; Auto reformatted panoramic measurements (the panoramic curve is automatically defined by Romexis ${ }^{\circledR}$ software) and Manual reformatted panoramic measurements (the panoramic curve is defined by the operator and then the new panoramic view will be automatically calculated by the Romexis ${ }^{\circledR}$ software). The slice thickness was 20.0 $\mathrm{mm}$ for the auto reformatted panoramic images and $25.0 \mathrm{~mm}$ for the manual reformatted ones.

Electric regimen settings used in panoramic and CBCT imaging enabled the observers to visually determine bony landmarks clearly, as determined by a series of similarly obtained radiographs with different exposure parameters from a pilot evaluation performed before commencing the current study.

\section{In all modalities, the following linear measure- ments were obtained}

Related to mental foramen: A vertical line was drawn perpendicular to the base of the mandible passing through the center of the mental foramen; measurements were made along this line.

i. From the inferior border of the mandible to the inferior $\&$ the superior borders of the mental foramen

ii. From the inferior border of the mandible to the superior border of the mandible.
Related to mandibular foramen: From the most superior radio-opaque point of the upper border of the mandibular canal, a vertical line was drawn from that point to upper and lower borders of the opposing mandibular bone and a horizontal line was drawn from that point to anterior and posterior borders of opposing mandibular bone. The vertical and horizontal lines are drawn perpendicular to each other and intersecting at that most superior radioopaque point.

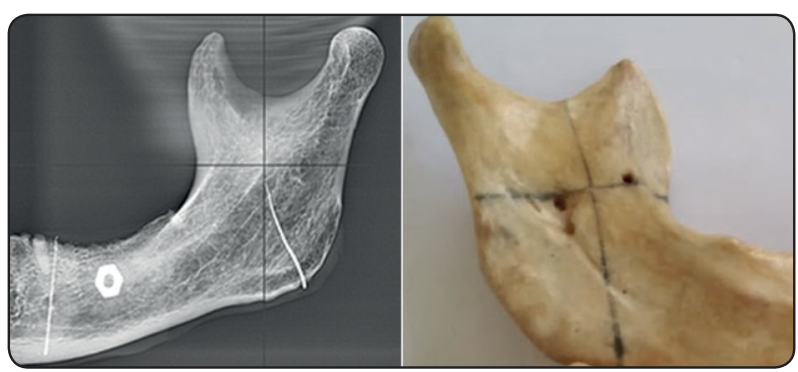

Fig. (1) Measurements related to mandibular foramen.

\section{Real direct measurements (Golden Standard):}

The pink set up modeling wax was removed from the mandibles' surfaces using hot water, ensuring smooth surfaces without any wax debris.

Linear measurements' lines were drawn on the mandible using a $0.5 \mathrm{~mm}$ pencil in a manneranalogous to the determination of linear measurements on the mandible using the software.

Mandibles were sectioned using a hack saw (Carrefour, China) in correspondence to the lines drawn on the mandibles surfaces'. The same researcher recorded all measurements after careful instruction from an experienced dentist. All recordings were made 3 times with the same vernier digital caliper (Facom ${ }^{\circledR}$, France) and an average was taken.

\section{Image Analysis:}

All images were assessed blindly and independently by two observers twice at two 
different sessions with a two-week period in between the two sessions to eliminate any inter and intra-observer variations.

The real measurements on the dried mandibles were performed 1 month after radiographic assessments. We intended to keep our sample specimens until all radiographic inter- and intraobserver measurements were completed, in order to avoid unexpected blurred landmark. The person responsible for the data analysis did not participate in the measurement sessions.

All images were analyzed using the same software, Planmeca Romexis ${ }^{\circledR}$ viewer (v 3.2.7), in HP pavilion dv6 notebook pc (Hewlett-Packard, Palo Alto, California, United States), with 15.6 inch HP LED screen with a resolution of $1366 \times 768$ pixels. The contrast and brightness of the images were adjusted using the image processing tool in the software to ensure optimal visualization.

Prior to the measurements of the anatomical landmarks, the observers were trained to use the Planmeca Romexis ${ }^{\circledR}$ viewer's (Planmeca Oy, Helsinki, Finland) measurement tools.

Radiographic measurements were made on the digital images using the specific software and then compared to the direct measurements obtained by using Facom ${ }^{\circledR}$ 1300e vernier digital caliper (Facom ${ }^{\circledR}$, France) with $0.01 \mathrm{~mm}$ precision. Direct measurements of the mandibles were considered the golden standard. All direct real and digital measurements were obtained in millimeters.

\section{Statistical analysis}

Numerical data were presented as mean and standard deviation (SD) values. Data were explored for normality by checking data distribution, calculating the mean and median and using Kolmogorov-Smirnov and Shapiro-Wilk tests. All data showed non-normal (non-parametric) distribution.
Wilcoxon signed-rank test was used to compare between different modalities' measurements and real measurements. It was also used to compare between right and left sides measurements' of the panoramic views.

Intra- and inter-observer agreements were assessed using Cronbach's alpha reliability coefficient, Intra-Class correlation coefficient (ICC) and their 95\% confidence interval (95\% CI). Cronbach's alpha reliability coefficient and ICC normally range between 0 and 1 . Cronbach's alpha value that is greater than 0.7 indicates "very good" agreement.

The significance level was set at $\mathrm{P} \leq 0.05$. Statistical analysis was performed with IBM ${ }^{\circledR}$ SPSS $®$ statistics version 20 for windows (Microsoft windows, Microsoft cooperation USA) (SPSS, Inc., an IBM company NY USA).

\section{RESULTS}

Intra- and Inter-observer agreement with Cronbach's alpha values:

\section{Intra-observer agreement:}

Digital Panoramic was "very good" (0.969 to 0.999); Auto reformatted panoramic was "very good" (0.912 to 0.988); Manual reformatted panoramic was "very good to perfect" (0.995 to 1.000).

\section{Inter-observer agreement:}

Digital Panoramic was "very good" (0.954 to 0.998); Auto reformatted panoramic was "very good to perfect" (0.995 to 1.000); Manual reformatted panoramic was "very good to perfect" ( 0.980 to 1.000).

No statistically significant difference - mostly tending to be underestimated - between linear measurements' means in all modalities used when compared to real ones except for "superior R.O. 


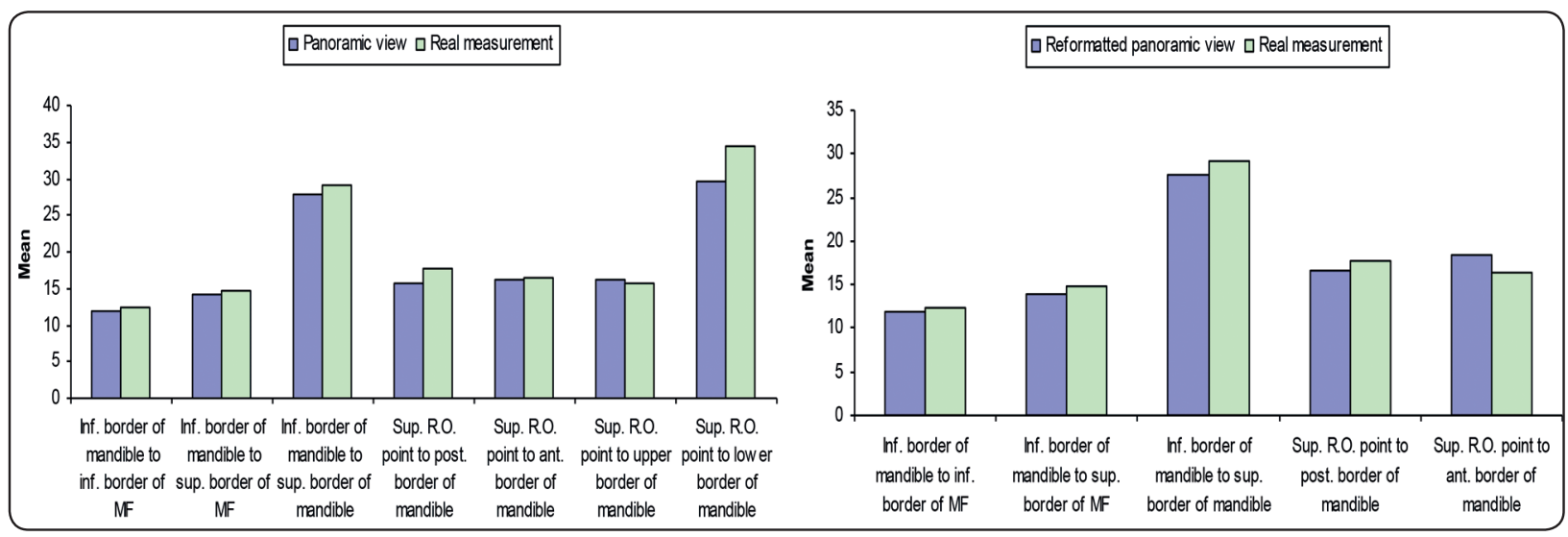

Fig. (2) Bar chart representing mean values of different measurements in panoramic (left), auto reformatted (right) view compared to real measurements

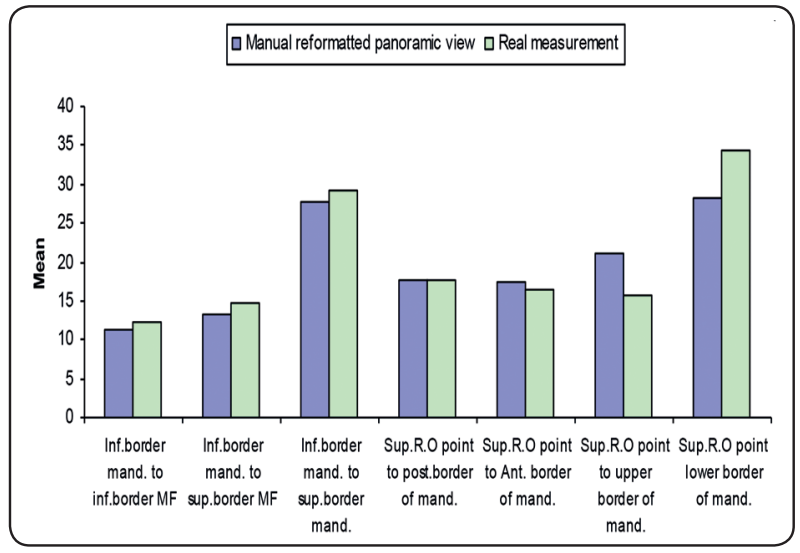

Fig. (3) Bar chart representing mean values of different measurements in Manual reformatted panoramic view compared to real measurements

point to the lower border of the mandible" which was significantly underestimated in all modalities used. Meanwhile, "superior R.O. point to the upper border of the mandible" was significantly overestimated in manual reformatted panoramic.

\section{Accuracy of imaging modalities for different lin- ear measurements:}

Inferior border of mandible to inferior border of MF: Auto reformatted panoramic showed the highest accuracy followed by panoramic view. Manual reformatted panoramic showed the lowest accuracy.
Inferior border of mandible to superior border of $M F$ : Panoramic view showed the highest accuracy followed by Auto reformatted panoramic. Manual reformatted panoramic showed the lowest accuracy.

Inferior border of mandible to superior border of mandible: Panoramic view showed the highest accuracy followed by Manual reformatted panoramic. Auto Reformatted panoramic showed the lowest accuracy.

Superior R.O. point to posterior border of mandible: Manual reformatted panoramic view showed the highest accuracy followed by auto reformatted panoramic. Panoramic view showed the lowest accuracy.

Superior R.O. point to anterior border of mandible: Panoramic view showed the highest accuracy followed by manual reformatted panoramic. Auto Reformatted panoramic view showed the lowest accuracy.

Superior R.O. point to upper border of mandible: Panoramic view showed the highest accuracy followed by Auto reformatted panoramic. Manual reformatted panoramic view showed the lowest accuracy.

Superior R.O. point to lower border of mandible: Panoramic view showed the highest accuracy followed by manual reformatted panoramic. Reformatted panoramic view showed the lowest accuracy. 


\section{DISCUSSION}

Panoramic view linear measurements' means showed no statistically significantly variation when compared to real ones, only one was significantly underestimated.

This finding is in agreement with many studies concluded that panoramic linear measurements were accurate; where, Kositbowornchai et al. (2007) stated that a strong positive correlation between the distances obtained with panoramic radiographs and dry mandibles has been reported. Vazquez et al. (2013) concluded that vertical measurements had acceptable accuracy and reproducibility when a software-based calibrated measurement tool was used. Peker et al. (2009) stated that vertical measurements are generally reliable.

On the other hand, we do not agree with Siu et al. (2010), Kjellberg et al. (1994) and Van Elslande et al. (2008) concerning linear measurements as they concluded that they were not accurate.

Many studies concluded that panoramic linear horizontal measurements were inaccurate; According to Peker et al. (2009) horizontal measurements were unreliable in panoramic radiography. Also Guler et al. (2005) believed that absolute horizontal measurements on a panoramic radiograph must be abandoned, because of the inherent image distortion and the un-linear changes in magnification. However the locations where horizontal measurements were taken were not clear. In our study there was no statistically significant variation between ramus horizontal measurements represented by "superior R.O point to the anterior and posterior borders of the mandible" in panoramic when compared to the real ones. Thus we do not agree with Guler et al. (2005) and Peker et al. (2009) concerning horizontal linear measurements.

Lee et al. (2012) believed that errors in available bone height on panoramic images are inevitable owing to the upward X-ray beam of a panoramic radiography machine, even if they are well taken with accurate positioning. In our study evaluation of bone height of the mandible is represented by "inferior border of mandible to the inferior and superior borders of the mental foramen and inferior border of the mandible to superior border of the mandible" which all showed no statistically significant variation when compared to real measurements. Thus our study is not in agreement with Lee et al. (2012).

In our study Auto and Manual reformatted panoramic showed no statistically significant difference - mostly tending to be underestimated - between linear measurements' means when compared to real ones except for "superior R.O. point to the lower border of the mandible" which was significantly underestimated in both modalities. Meanwhile, "superior R.O. point to the upper border of the mandible" was significantly overestimated in manual reformatted panoramic. Up to our knowledge there were no studies on linear measurements performed on Auto and Manual reformatted panoramic, and compared to real physical measurements.

It was not clear whether previous studies on the reformatted panoramic were Auto or Manual reformatted type; however Ludlow et al. (2006) found that CBCT (2D) technique provided acceptably accurate measurements of mandibular anatomy when compared to physical measurements. On the other hand, Angelopoulos et al. (2008) stated that the inherent inaccuracies of the CBCT reformatted panoramic images have prompted only a few studies to compare the accuracy level of these reconstructions not only with conventional images but with the true anatomy by direct measures. Ludlow et al. (2007) found that the panoramic reconstruction followed the curvature of the mandible resulting in linear measurements on the image to be overestimated, in a study where dried skulls were scanned to determine vertical and horizontal length accuracy when reconstructed in to panoramic projections. 
Radiographic measurements related to ramus are represented by 2 vertical measurements' means; superior R.O point to the upper \& lower borders of the mandible and 2 horizontal measurements' means; superior R.O point to the anterior \& posterior borders of the mandible. No statistically significant variation between Ramus horizontal measurements in digital panoramic, auto and manual reformatted panoramic and the real ones. "Superior R.O point to lower border of mandible" was significantly underestimated in the three modalities and "Superior R.O point to upper border of mandible" was significantly overestimated in Manual reformatted panoramic. Our study is not in agreement with Turp et al. (1996) who found that the panoramic radiographic measurements related to the condyle and ramus heights were found to be poorly correlated to direct values in dry mandibles.

\section{CONCLUSION}

Comparable results were shown when linear measurements' means from Digital panoramic, CBCT derived Auto and Manual reformatted panoramic were compared to real ones. Radiation dose to patients must be concerned.

\section{REFERENCES}

1. AL-Nakib L. Magnification in panoramic radiography. $\mathrm{J}$ Bagh Coll Dentistry 2005; 17(3); 45- 47.

2. Angelopoulos C, Thomas SL, Hechler S, Parissis N, Hlavacek M. Comparison between digital panoramic radiography and cone-beam computed tomography for the identification of the mandibular canal as part of presurgical dental implant assessment. J Oral Maxillofac Surg. 2008; 66:2130-2135.

3. Devlin H, Allen PD, Graham J, Jacobs R, Karayianni K, Lindh $\mathrm{C}$, et al. The role of the dental surgeon in detecting osteoporosis: the OSTEODENT Study. Br Dent J 2008; 204: E16.

4. Guerrero ME, Jacobs R, Loubele M, Schutyser F, Suetens $\mathrm{P}$, van Steenberghe D. State-of-the-art on cone beam CT imaging for preoperative planning of implant placement. Clin Oral Investig 2006; 10:1-7.
5. Guler AU, Sumer M, Sumer P and Bicer I. The evaluation of vertical heights of maxillary and mandibular bones and the location of anatomic landmarks in panoramic radiographs of edentulous patients for implant dentistry. Journal of Oral Rehabilitation 2005; 32; 741-746.

6. Hutchinson SY. Cone beam computed tomography panoramic images vs. traditional panoramic radiographs. American Journal of Orthodontics and Dento-facial Orthopedics 2005; 128:550.

7. Kjellberg H, Ekestubbe A, Kiliaridis S, Thilander B. Condylar height on panoramic radiographs. A methodologic study with a clinical application. Acta Odontologica Scandinavica 1994; 52:43-50.

8. Kobayashi K, Shimoda S, Nakagawa Y, Yamamoto A. Accuracy in measurement of distance using limited conebeam computerized tomography. Int $\mathrm{J}$ Oral Maxillofac Implants 2004; 19: 228-231.

9. Kositbowornchai S, Siritapetawee M, Damrongrungruang T, Khongkankong W, Chatrchaiwiwatana S, Khamanarong $\mathrm{K}$ and Chanthaooplee T. Shape of the lingula and its localization by panoramic radiograph versus dry mandibular measurement. Surg Radiol Anat 2007; 29: 689-694.

10. Langlois CO, Sampaio MCC, Silva AER, Da costa NP, Rockenbach MIB. Accuracy of linear measurements before and after digitizing periapical and panoramic radiography images. Braz Dent J 2011; 22(5):404-409.

11. Lee SM, Lee SS, Huh KH, Yi WJ, Heo MS and Choi SC. The effects of location of alveolar crest on the vertical bone heights on panoramic radiographs. Dentomaxillofacial Radiology 2012; 41, 117-121.

12. Ludlow JB, Davies-Ludlow LE, Brooks SL, Howeron WR Dosimetry of 3 CBCT devices for oral and maxillofacial radiology: CB Mercuray, NewTom, 3G and i-CAT. Dentomaxillofac Rad 2006; 35:219-26.

13. Ludlow JB, Laster WS, See M, Bailey LJ, Hershey HG. Accuracy of measurements of mandibular anatomy in cone beam computed tomography images. Oral Surg Oral Med Oral Pathol Oral Radiol Endod 2007; 103(4):534-542.

14. Mischkowski RA, Ritter L, Neugebauer J, Dreiseidler T, Keeve E, Zoller JE. Diagnostic quality of panoramic views obtained by a newly developed digital volume tomography device for maxillofacial imaging. Quintessence Int 2007; $38: 763-772$

15. Nakagawa $Y$, Kobayashi $K$, Ishii $H$, Mishima $H$, Ishii $K$, Asada K, et al. Preoperative application of limited cone beam computerized tomography as an assessment tool 
before minor oral surgery. Int J Oral Maxillofac Surg 2002; 31(3):322-26.

16. Park JB. The evaluation of digital panoramic radiographs taken for implant dentistry in the daily practice. Med Oral Patol Oral Cir Bucal 2010; 15: e663-666.

17. Pawelzik J, Cohnen M, Willers R, Becker J. A comparison of conventional panoramic radiographs with volumetric computed tomography images in the preoperative assessment of impacted mandibular third molars. J Oral Maxillofac Surg 2002; 60:979- 984.

18. Peker I, Gungor K, Semiz M and Tekdemir I. Localization of Mental and Mandibular Foramens on the Conventional and Digital Panoramic Images. Coll. Antropol. 33 (2009) 3: 857-862.

19. Pittayapat P, Galiti D, Huang Y, Dreesen K, Schreurs M, Couto Souza P, Rubira-Bullen IRF, Westphalen FH, Pauwels R, Kalema G, Willems G and Jacobs R. An in vitro comparison of subjective image quality of panoramic views acquired via $2 \mathrm{D}$ or $3 \mathrm{D}$ imaging. Clin Oral Invest 2013; 17:293-300.

20. Scarfe WC, Farman AG and Sukovic P: Clinical Applications of Cone-Beam Computed Tomography in Dental Practice. J Can Dent Assoc. 2006 72(1):75-80.

21. Schropp L, Alyass NS, Wenzel A, Stavropoulos A. Validity of wax and acrylic as soft-tissue simulation materials used in in vitro radiographic studies. Dentomaxillofacial Radiology 2012; 41: 686-690.

22. Schropp L, Stavropoulos A, Gotfredsen E, Wenzel A. Calibration of radiographs by a reference metal ball affects preoperative selection of implant size. Clin Oral Investig 2009; 13: 375-381.

23. Siu AS, Chu FC, Li TK, Chow TW, and Deng FL. Imaging modalities for preoperative assessment in dental implant therapy: an overview. Hong Kong Dent J 2010; 7:23-30.

24. Tohnak S, Mehnert A, Crozier S, Mahoney M. Synthesizing panoramic radiographs by unwrapping dental CT data. Conference Proceedings: Annual International Conference of the IEEE Engineering in Medicine and Biology Society 2006; 1:3329-3332.

25. Turp JC, Vach W, Harbich K, Alt KW, Strub JR. Determining mandibular condyle and ramus height with the help of an Orthopantomogram-a valid method? Journal of Oral Rehabilitation 1996; 23:395-400.

26. Van Elslande DC, Russett SJ, Major PW, Flores-Mir C. Mandibular asymmetry diagnosis with panoramic imaging. American Journal of Orthodontics and Dentofacial Orthopedics 2008; 134:183-192.

27. Vazquez L, Nizamaldin Y, Combescure C, Nedir R, Bischof M, Dohan Ehrenfest DM, et al. Accuracy of vertical height measurements on direct digital panoramic radiographs using posterior mandibular implants and metal balls as reference objects. Dentomaxillofac Radiol 2013; 42: 20110429.

28. Wakoh M, Nishikawa K, Otonari T, Yamamoto M, Harada T, Sano T, Ooguro T. Digital subtraction technique for evaluation of peri-implant bone change in digital dental imaging. Bull Tokyo Dent Coll. 2006 May; 47(2):57-64. 